\title{
LES CHAUSSÉES DE REIMS ET DE TONGRES EN PAYS NERVIEN
}

\author{
par Jean-Louis BOUCLY
}

Du chef-lieu de la Cilé des Verviens, Bavai. rayonnaient sept chaussées d'inégale importance (fig. 1). Parmi elles, celles de Reims el de Tongres jouirent un ròle de tout premier ordre. Sur le tracé particulier de res voies. sur leur structure el sur les bourgs auxquels elles donnèrent naissance en terriloire nervien. nous avons fait plusieurs remarques nouvelles ${ }^{1}$ que nous voudrions exposer dans cet article.

\section{Le tracé de la chaussie de Reims.}

Lne des particularités les plus frappantes des voies romaines du . Nord de la ciaule est la rectilude de leur tracé. Négligeant en effel les accidents du terrain, elles ront, selon l'expression d'l. Will2, "droit au but". A cet égard, celles de Vermand, de Trieves el de Cologne sont, sur toule l'étendue du lerritoire nervien, exemplaires. On a mème accoutumé de ranger parmi ces chaussées rectilignes celle de Reims dont les inflections n'apparaissent pas sur une carte a grande

l Lin fait, cet article est la synthese do plusieurs recherches restees inedites ot menies depuis 1965 avec l'etroite collaboration de R. Jolin. C'est lui qui a dessiné tous les plans que nous avons reproduits en les completant au besoin et cest pour nous un agrabable devoir de lui temoigner, une fois encore, toute notre gratilude.

2 F. Wis., Isavai, cilé gallorromaine, Douai, 1957. échelle ${ }^{3}$. Or, une étude plus précise de son tracé révele plusieurs anomalies.

On constate en eflet que, de la frontière sud de la civilast jusqu'a Bavai, elle forme une ligne brisée composée de quatre segments inégaux et, surtout, qu'a hauteur de Larouillies, elle abandonne brusquement la direction du nord qu'elle suivait depuis Vervins pour obliquer vers l'ouest et gagner Bavai. (Or. si l'on prolonge au nord de Iarouillies l'axe de la chaussée, on atteint litroeungt, ${ }^{5}$ puis l'oppi-

3 . . Mкитюхs, Les roules romaines de la Belgique, dans Industrie, IX, 1955, no 10; J. Mentexs avec la collaboration d'A. Inspr-Mryse, la Belgique à l'ipoque romaine, Cartes archéologiques de la Belgique 1-2, Sorvice National des Fouilles, Bruxelles, 1968, fig. II, p. 20. La plupart des aul res cartes publiés récemment reproduisent les précedentes : A. Waxkexise, La Belyique à l'́poque romaine. Siles urbains, villageois, religiens et militaires, centre National do la recherche archeologique en Belgique, seric K, HI, Bruxelles, 1972, plan I; 13. Bntı.к, La defense du terriloire an BasEmpire, dans La Belgique de César à C:lwvis, lossirers de. l'Archéologie, n"21, 1977, p. 100)-101.

+ (i. Fandr-Figrmass, les limiles de la cilí des verviens, dans $l$ Antiquili rlassique. XXI, I9:2, p. $33 x-35 x$

5 Ies anciennes formes du nom d'Elroemet : Struen 1150, , Estruen $1165,11 \times 6$ sont à rapprocher d'Estrun Cord et Pas-de-talais oil se trouvent les restes de deax oppida nervien et atrobate. Vin toul cas, Ir site convient parfaitement sinon à un oppidum, du moins à un caslellum qui aurail surveille la vallow de 


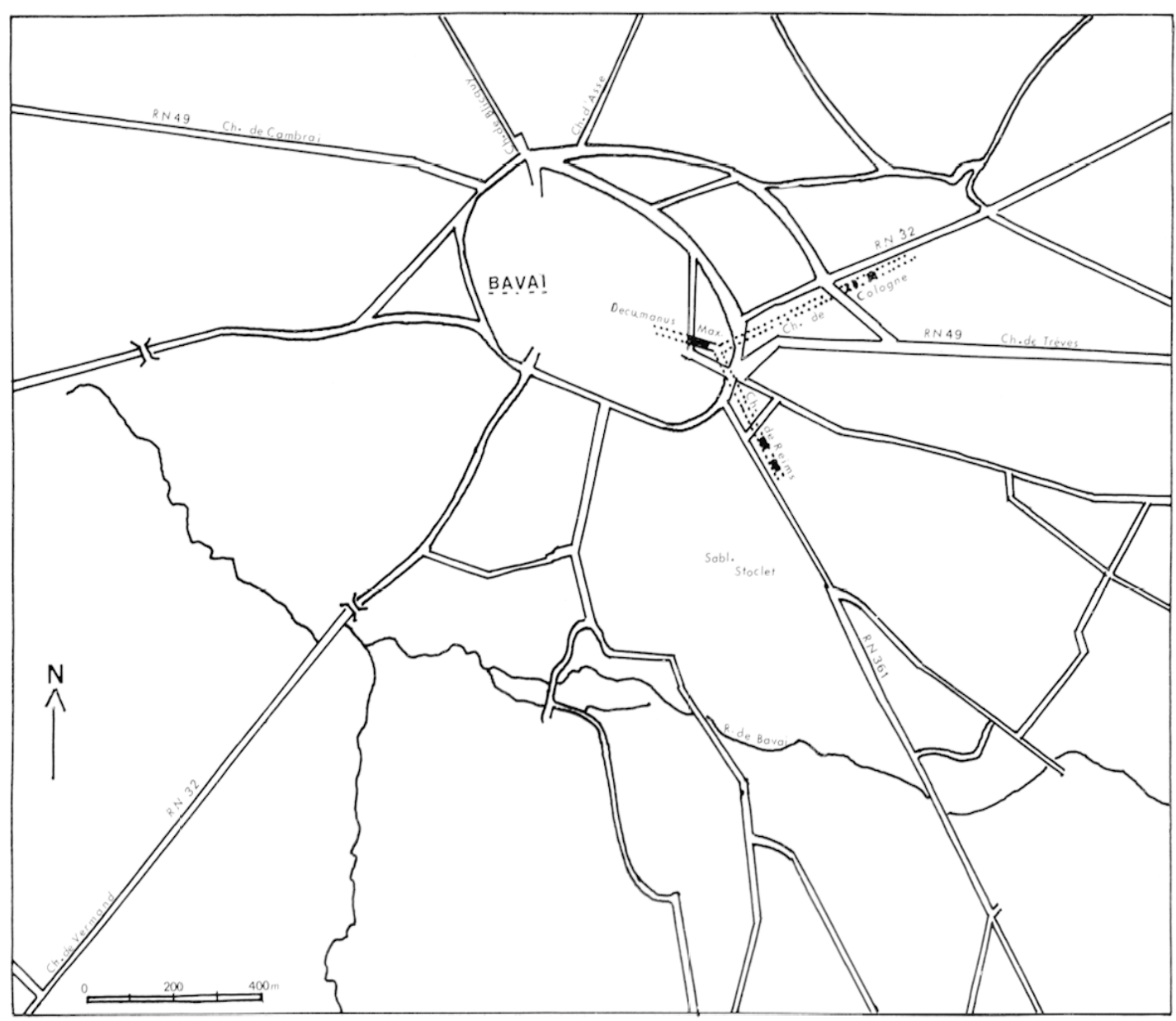

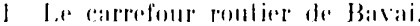

dum d'Aresnelles, puis, par Maubeugé, eelui de Rouveroy se dessine ainsi le trace d'une

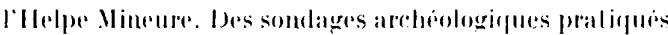
en divers endroils du plateant permettraient pent-ìtre

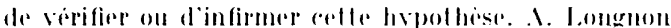
Les noms de lien de la lirance. 192019-19:9, p. :13, fait

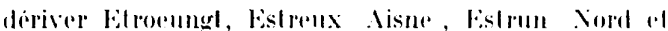
Pas de Calais du nom germantque stroom designant un cours-d'antu.

6 I.es mommates celliques decomverles a llaubengu

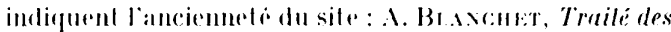

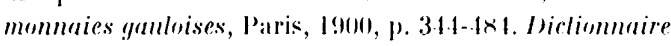
archologique de la tianle, II, p. 17I. H. DE I A ToLk, Allas des monnaies ganlonises, paris, I $\times 992, n^{\prime \prime s} \times 6.37$,

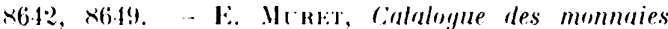
ganloises de la Bibliolheque Nalionale, Parjs, l $\times x ?$, II"* $\times(637, \times 6.4: \times 6.19$.

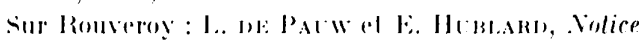

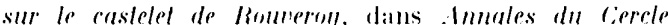

piste relt ique diase nord-sud comme celui de la R.X. 2 qui. de nos jours, suit partiellemenl la mème directrice gróg graphique fig. 2 .

Linsi donce la chaussée de heims semble avoir emprunté. en le rectifiant au besoin. le tracé d'une ancienne piste gauloise qu'elle abandonnait a hauteur de larouillies pour gangner le rhef-lieu de la Cilí. Baviai. nouvelle-

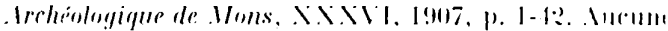

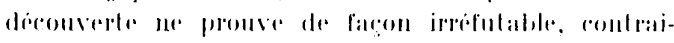

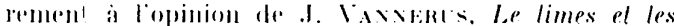
fortificalions gallo-romaines de Belgique. enquile lopu-

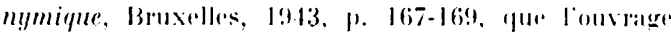

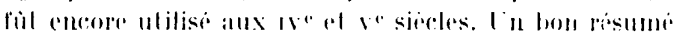

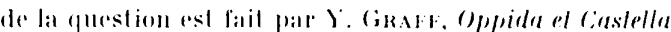

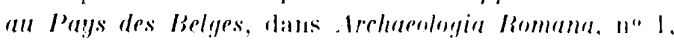

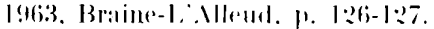




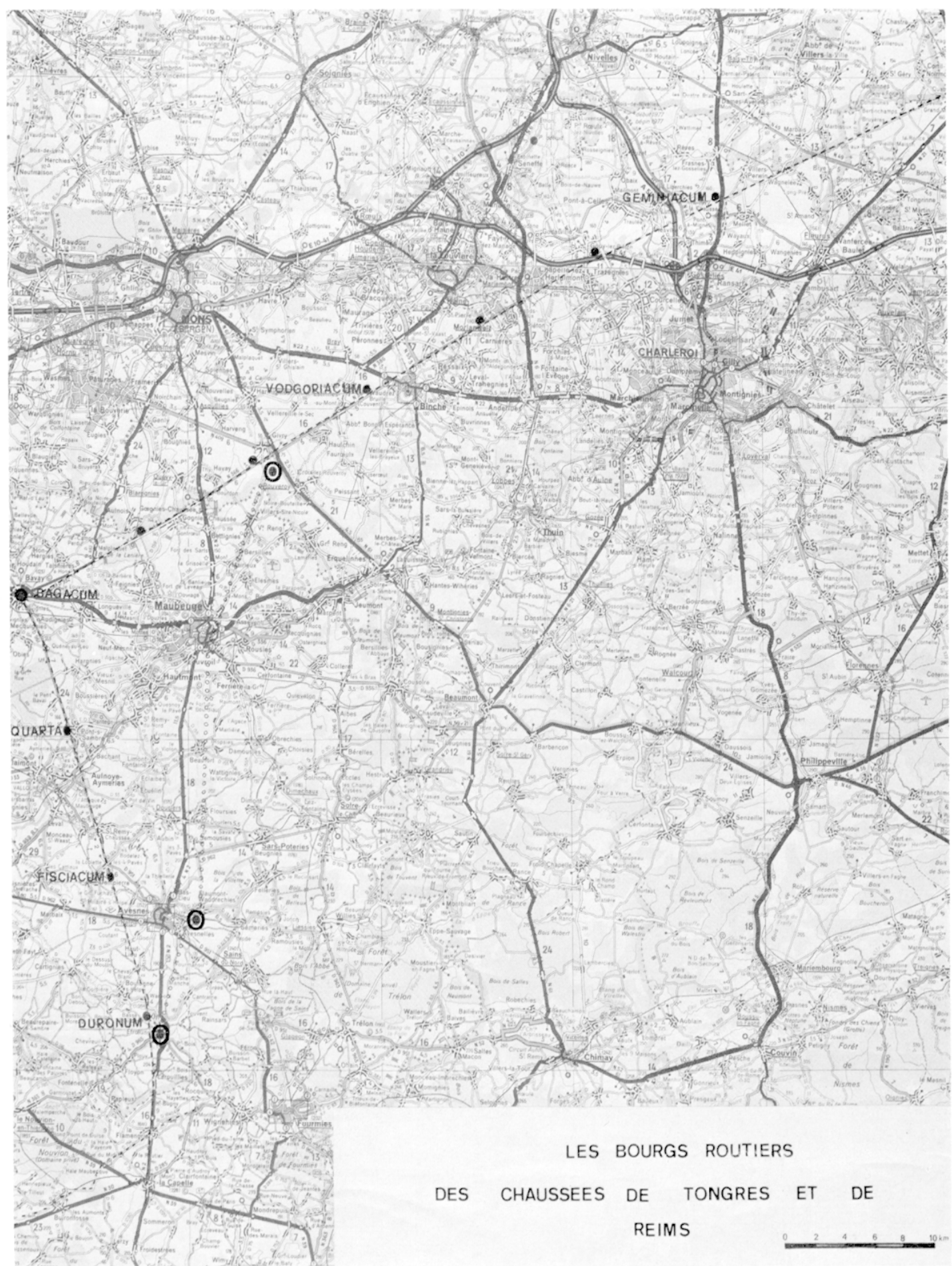

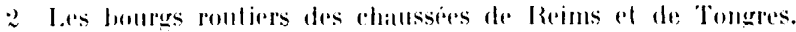


ment (rée 2) km au n.-o. d'Avesnelles. dans une région de plaine et sur un site mieux adapté aux conceptions romaines. Le cas d'une ancienne piste celtique ainsi corrigée par les ingénieurs romains n'est pas rare en Gaule. Deux exemples suffiront a l'illustrer : Bibracte. abandonné en faveur d'Aulun, situé $20 \mathrm{~km}$ a l'est, el, plus pres de nous, Pommiers. délaissé pour soissons? Chaque fois, on s'apercoit que la ou les voies romaines, dirigrées de loin vers l'ancienne place-forte, se détournent a un endroit donné pour aller desservir la nouvelle ville.

Quoi qu'il en soit, il est presque impossible de déterminer pour quelles raisons précises l'ingénieur romain choisit le tracé en ligne brisée du tronçon de roule qu'il devait frayer de Larouillies a Bavai. sans doute, la construction des chaussées et l'établissement du cadastre furent-ils étroitement liés ou celles-lá servirent-elles souvent de base à celui-ci. En tous cals. ces travaux délicats d'arpentage durent se faire en utilisant des points de reperes que nous ignorons. Dans ces conditions. nous ne pouvons dire si c'est par hasard ou a bon escient que le troncon terminal de la voie est orienté vers l'oppidum d'Avesnelles. T'out ce que nous pouvons supposer c'est que l'ingénieur romain dut lenir compte des trois rivières que la route avait à franchir: les deux Ilelpe Majeure et Mineure, et la Sambre, éviter les fonds de vallées marécageux. choisir les endroits les plus facilement guéables tout en conservant le plus longtemps possible la mème direction. Ln outre. c'est aux points hauts de Larouillies, de Boulogne. du Quesneau-Leu que les changements d'orientation se constatent, ce qui laisse supposer que, de ces endroils. des visées furent failes de proche en proche pour jalonner le tracé de la voie (fig. 3).

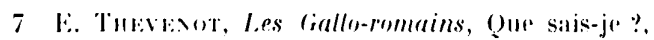
Paris, 19599, p. 17, … P. I.smax, at propos di une coupe de boie rumaine en forit de Compiegne: Ia boie de sentis à soisssms, dans Cahiers archónlogiques de Picardie,

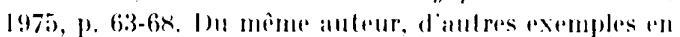

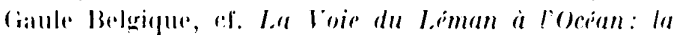
tranche orienlale, ital de la question el propositions nourelles, dans Cacsurudunum. 1975), n" 11, p. 10:-111x.
Struclure de la chanssée à la sorlie de Bavai.

De la chaussée de Reims nous vimes en coupe l'empierrement en 1968, dans la parcelle 1:3413, aul cours des travaux de terrassement nécessités par la construction du château deau ${ }^{8}$ (fig. 4).

sous 0,45 in de lerre végétale. nous observàmes un cailloutis de $0.30 \mathrm{~m}$ d'épaisseur, reposant sur une couche de pierrailles de calcaire bleu, tassees dans du sable sur une hatuteur de $0.30 \mathrm{~m}$ environ. Cet empierrement. de $5,80 \mathrm{~m}$ de largeur. s'étendait sur un remblai de limon jaune dont la base mesurait $12.80 \mathrm{~m}$ du nord au surd. sur les còtés de la chaussée, s'apercevait un lit de marne mauve. Peut-ètre cette couche empèchait-elle la remontée de l'argile lout en assurant le drainage des eaux d'infiltration vers les fossés latéraux. Elle devait probablement recouvrir ce quil restait de l'empierrement el servir d'assise à la couche de roulement disparue (fig. 5).

Au nord el au sud de l'empierrement principal. deux autres semblables, mesurant respeclivement 4.70 el $4 \mathrm{~m}$ de largeur. s'étendaient au mème niveau que le premier, sur les boues grises déposées par les paux de ruissellement. Quelque $30 \mathrm{~m}$ plus au sud. dans la parcelle 134: 1 , une autre fouille, mais peu profonde? nous permit de constater que. seul. l'empierrement le plus oriental subsistait en conservant la même strurture (fir. 4). Ainsi, la tranchée du chàteau d'eau révéla-l-elle la coupe d'un tronçon urbain de la chaussée de Reims dont le tracé dul varier dans les faubourgs de Bavai comme celui du decumanus auquel la voie romaine aboutissait ${ }^{10}$.

8 Je cas decomvertes, H. Bieveled a rendu partiellemont comple dans Vole sur le premier mille de In Barai-Reims. dans Revue du . Vord. I.I. 11" 20:, 1969, p. 164-166. I a photo publier a la ligure I montere dans

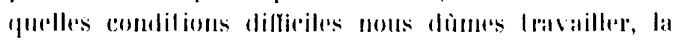
betonnense itant installie a laplomb des vestiges antiyues.

9) La fouille ful entreprise an aveil 1966, dans la

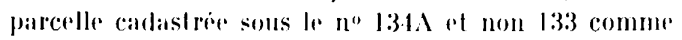
l'indique H. Bıtrat.:T, op. cil.. p. 464. Jans la parcelle contigua, no 133, la roule ful recoupere partiellement an

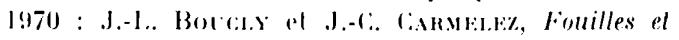
deconuerles en dehmrs du "Cirand-linsemble ", dans

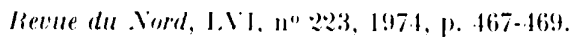

lo J) ae decumants, un premier troncon ful observi par H. bievelel, dans la ruelle Jean-eiharles, 

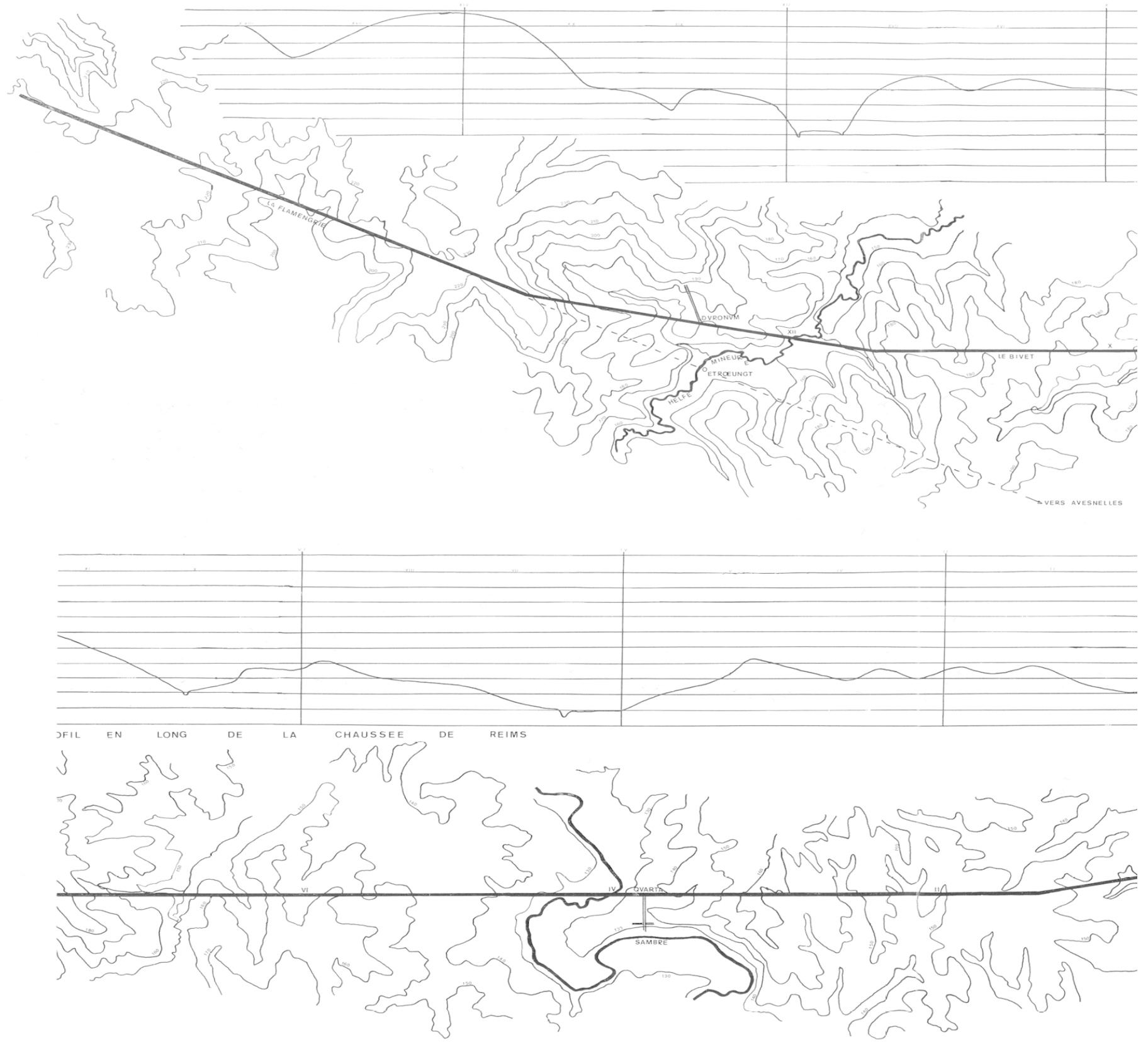

3 Profil en long el trace de la chaussece de Reims sur le lerrituire de la cite des Nerviens. 


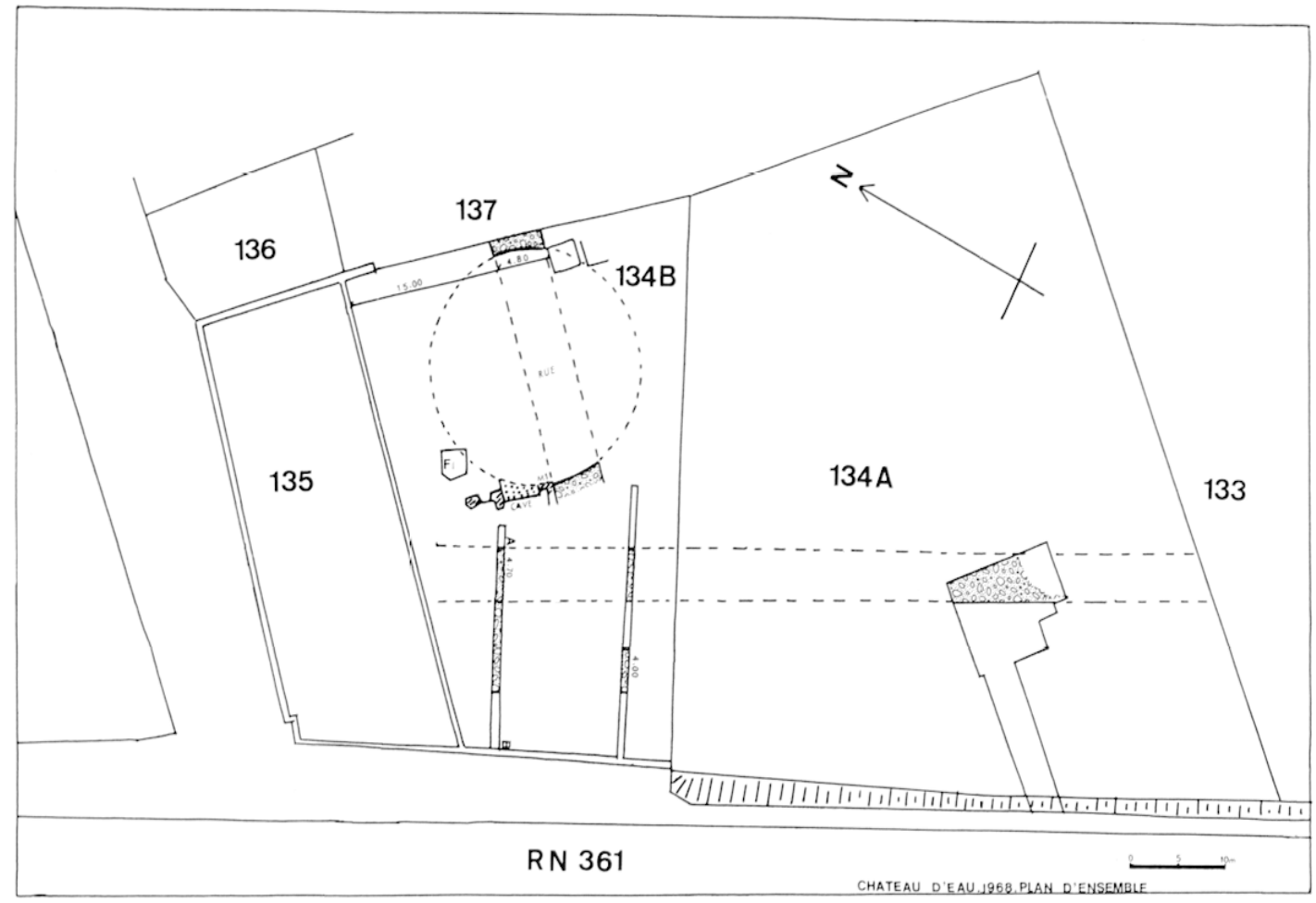

4 Ja chanssce de Reims. Plan de siluation des foulles de l96r.

Quoi qu'il en soit, à l'ouest de l'empierrement principal. sous quelque $1.35 \mathrm{~m}$ de terre végétale el de boues, et sur $: 3,20 \mathrm{~m}$ de largeur,

en 1961: 1 la ruelle .lean-1:harles, daus Annales du Cercle Archoblogique de Mons, 64, 195x-1961, p. 57-6x, pl. Il et fig. 3. Fin 1965, un second troncon apparul plus a leest, dans la parcelle no 74 , lors des I ravaux prealables a la conslruction de la cite des bouanes. be ces découvertes, nous rendrons comple dans un prochain article. Comme nous l'indiquons sur le plan qui montre le profil en long do la chausser de Reims, les rues romaines ont, a l'est en a l'onest de la cilo antique, une orientation differente. Cefte anomalie s'explique probatsement par le fait que te plan d'urbanisme inilialement preva ne fut qu'en partie réalise sous clande. I. irbanisation du secteur oriental ne daterail que du debut de lipodpe favienne, comme la construction dans celle partio de la ville du château d'eatu of des thermes J.-L. Boccis, In fragment

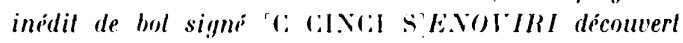
dans les ruines de I Aqueduc de Barai, a saint-Rrmydu-.Vord, dans liallia, 35), 1977, 1. 263-269. Enfiu, apres leincendie de Bavai, vers la fin du lae siectre des rues nouvellement fravers couperent certaines insulae anterienres a peu pries selon leurs diagomales. nous vîmes les restes de la chaussée primitive, réduits à une épaisseur de $0,20 \mathrm{~m}$ environ et consistant en une simple couche de petits silex noirs (figr. Ђ). Cette découverte n'est pas sans rappeler celle que fit, le 4 mars 1926. J. Quévy, dans la parcelle B2 2:33) oi il mit au jour, sous $0,70 \mathrm{~m}$ de terre noire et de pierres. une partie de l'empierrement de cetle chaussée. consistant également en une simple couche de silex de $0.20 \mathrm{~m}$ d'épaisseur ${ }^{11}$. Telle était aussi la structure des routes de Trives el de Tournai ${ }^{12}$ que nous vîmes en coupe en 1969 a llaubeuge.

11 Pro Nervin, 111, fo liv., Mresmes, 1927, 1) 430

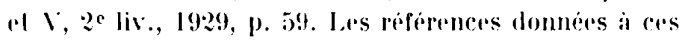
articles par H. Butvist, Vote sur le premier mille de la Bavai-Reims, op. cit., p. 46:, sont frronérs.

12 II sagit an fait du troncon commmu alux

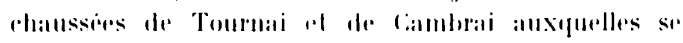
reliaient des voles venant de Famars : G. Brost al II. INVirzatit, Le castellum de Fanum Marlis IFamars. Nord), dans fiallin, XIX, 1961, p. 162. 
au lieu-dit L'()uvrage ${ }^{13}$, et en 1967 a La Perche, sur le territoire de Saint-Waast-la-Vallée

Aucun objel, malheureusement, ne permil de dater les empierrements sucressifs de la chaussie de Reims. Cependant, au cours de la fouille de sauvelage du chateau d'eau. nous décourrimes des lessons de vases arétins et observames en coupe, au nord du fond de cabane F1, une grande fosse de $3 \mathrm{~m}$ environ de profondeur, comblée par du limon jaune auquel se mèlaient des fragments de grands vases ayant l'aspect du liège. Si nous n'eumes pas le temps de dessiner le plan ni d'explorer les restes de ret habitat intéressant ${ }^{15}$, nous fimes cependanl d'autres trouvailles, a l'est du mur III d'une cave romaine, qui atlestent l'ancienneté de l'occupalion aux abords immédiats de la chaussée (figg. 4). Nous mîmes en effet au jour des tessons de vases façonnés a la main ${ }^{16}$, un petit godel grossier et a peine cuit ${ }^{17}$ ainsi que plusieurs objets en bronze. Parmi eux, il nous faut signaler un fragmenl de bracelet dont le jonc s'ornait de grappes de nodosités, analogue à celui exposé au Musée ${ }^{18}$ el date. d'apris les trourailles de Bajc SWlovaquie) et d'Ilvesheim (Bade-Wurtembera, de la fin du ${ }_{11}{ }^{\mathrm{e}}$ et du début du $\mathrm{I}^{\mathrm{e}}$ siécles av. J.-C.. ${ }^{19}$. Ajoutons enfin que la mème époque pourrait ètre proposíe pour l'urne rinéraire situliforme, arénée sous le bord. déeouverte

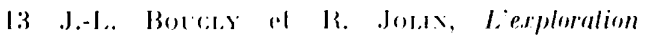
archeologique de la wie Banai-Trines, dans Kerue du Nord, 1.1. 1, n'2:3, 1974, p. 461-165.

1.4 H. Birentel a rendu comple de contle decourepte: 1u village de Lomvignies, dans Ilommayes à . M. Renard, Lalomus, 103, 1969. p. 44, appendice :2.

15 Cefte deconverte inedite fut faite par R. Jolin et moi-mème un dimanche soir, apres la fonille de la

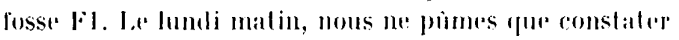
la disparition des restiges, fo tron ayant ble hetommi sans que nous en enssions ild arertis.

16 Fragments numboties $6 \times \%$ 25.1, 272.1, 289.1. leux-ci, comme les pieces suivantes, ne sonl pas accessil,les pour loinstant, en raison du lansfert des collections dans le nouveau llaser ef de leur reclassement en cours.

$176 \times \% 253: 1$

is $6 \times 2 \% .35$.

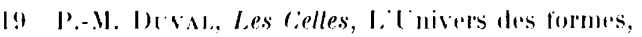
P'iris, 1977, p. 1333, tiq. 13:2. non loin de la, pris de la chaussée, dans la sabliere storlet ${ }^{20}$.

Les bourgs Lrarersés par la chanssé de Reims.

Maris les traces d'ocrupalion relevées aux abords de la chaussée, sur loute l'étendue de l'arrondissement d'dresnes. sont loin d'ètre ausisi anciennes. lilles ont permis cependant de lonaliser aver cerlitude lrois vici silues. a des intervalles réguliers de quatre lieues. soit six milles romains (fig. 3 ) : Quarta, press de lat sambre. Fisciacum. dont le nom évoque le fiscus impérial ${ }^{21}$ el rendu célébre par l'oraloire et les miracles d'un religieux irlandais, mort a Fuchau en biot, saint Etton ${ }^{22}$, el, enfin. Duronum, la seule des trois bourarades a itre signalée par les ilinéraires antiques ${ }^{23}$. Une répartition aussi régulière de lhabitat ne peut ètre due au hasard. Jussi, peut-on penser que res rici se developperent a partir des relais créés par l'administration romaine pour assurer le bon fonctionnement du cursus publicus. Ainsi, il y aurait eu un relais (mulalio) toutes les quatre lieues el. probablement, une étape (mansio) loutes les doure lieues. soit $26,664 \mathrm{~km}$. La seule qui existal en territoire nervien devail àtre Inuromum que l'Itinćraire d'Antonin silue effectivement a XII lieues de Bavai24.

Gependant, comme il est naturel de le constater pour des aggolomérations virantes. ces bouras ruraux se developperent dhacun a sa maniere, oblitérant au fil des siecles le caractere arliliciel des premiers établissements

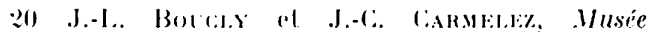
Archeologique de Bawai, (indele provisoire, 1976, p. 11, 1106.

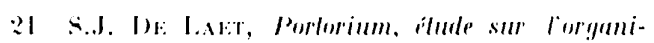
salion donaniore chez les lommins, surloul à léporgue du Ilaul-Empire, Bruges, 1949. R. CH1:valdus, Les mos romaines, P.I .F., l'aris, 1972, chap. 10, I.a Loponymir des voles antiques, p. 1.13-1.17.

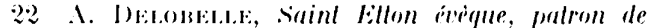
lompierre, Bar-1r-1)uc, 3" ind., 1 x92.2.

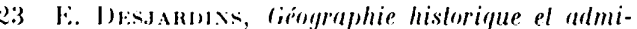
nistrative de la Cimble romaine, l'aris, 1876-1893, IV,

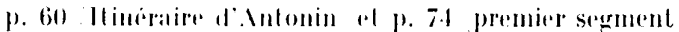
de la Table de Prolinger.

2.4 L. Drsonthas, op, el loce cil. 


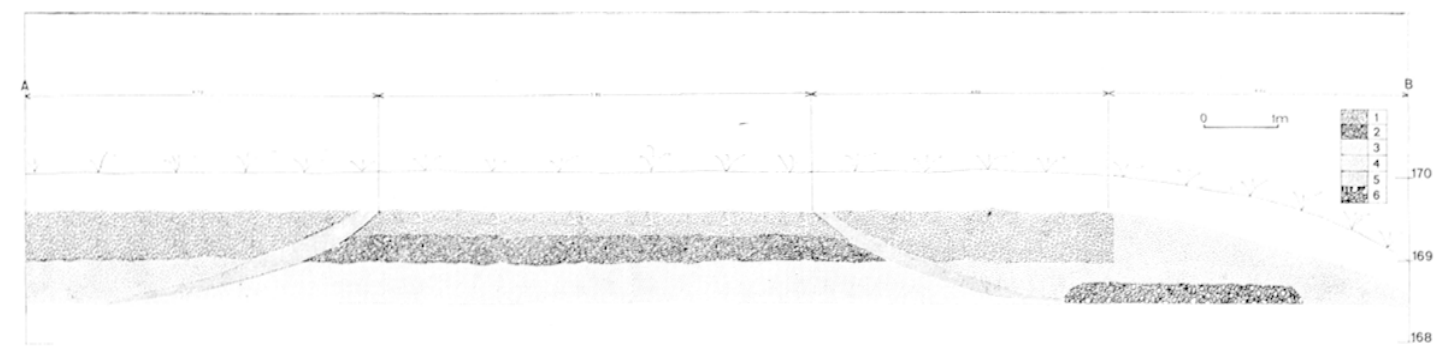

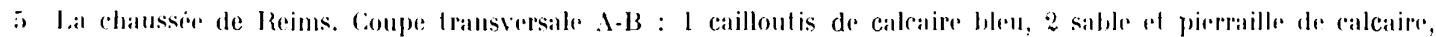
3 limon jaunr, 1 boures grises, 5 marue violetle, 6 petils silex noirs.

arministratifs qui avaient favorisé leur apparition. Aussi présentent-ils lous l'aspect de villages-rues, egrenanl leurs constructions le long de la chaussée et concentrant leurs principaux batiments en un rentre urbanisé d'ou partent généralement, vers d'autres élablissements ruraux, des voles secondaires ${ }^{25}$. Ainsi, celui de Quartes s'élendail-il au moins jusqu'a Pont-sur-siambre 26 . elui de Fuchau a-t-il laisse des traces nombreuses depuis le Vilers jusqu'au grué de l'Helpe ${ }^{27}$ el celui de

25) I Ouarles, somblanchait sur la chansser mu rue dont on vil des troncons en 1892 ch. linon, l'Avesnois próhishorique, gaulois, grallo-romain el franc,

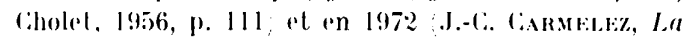
cili des Verviens: inventaire archeologique des confins móridionanx, 2 vol. daclylographies, thise inedite, J.ille, 1973, II, 1. 34-35. Non loin de l'eglise, nous remarquames, en 1972, la coups: d'une autre rue, anparemment perpendiculaire a la precódente, dams le latus nord de la route que l'on chargissail

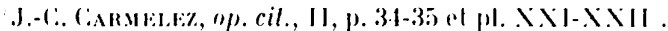
A Elroenungl, un diverticulum se dirigeant rers 1. vouvion ful parliellement mis an jour en $1 \times 60^{\circ}$ E. Marox, Misloire d'Elroeungl, Paris, $1 \times 62$, p. 2X. Nous en vimes une aul re coupe ('n 1975): J.-1.. Boter. Guelques dicouvertes forluites à Etroeungl el a Larouillies, dans Revue du . Vord, I.VIII,1976, p. 6×1.

26 Aux decouvertes signalés par th. liroj op. cil., p. 109, il faul ajouler celles de debris de fours de potiers ol do depotoirs: R. I)E.Manne, les morliers de I'onl-sur-sambre el de lalelier de Brarialus, dans septentrion, 2, fasc. 11-12, 1972, p. 16-5)t. I a trouvaille dale du mois doctobre 1970. Nous avons continue la fouille asec J.-C. larmelez op. cil., I, p. 346. II sagissail d'un depotoir de V.Mklatis, et non de BR.ARI.ITISt, comme l'avait cru llefmatre. D'autres travalux, en novembre 1970, amenerent la decomverte de lessons semblables pres de la tour de gurel :

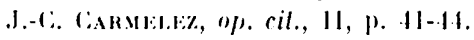

27 sur ces decomvertes, th. Croix op. cit., p. 1151:20, a donne un bon aperen.
Mnromum-lilroeungl, bien que son rentre fìt situé a la Pairée, avait-il des écarts a Basse-Boulogne et a Warpont ${ }^{28}$. Quoi quil en soit. il est intéressant de constater que res aggomérations ne se rencontrent, que le long d'une parlie seulement de la chaussée, celle précisément qui traverse le territoire nervien el qui est la plus rectiligne. Iu rontraire. Iorsque le tracé de la voie romaine, chez les Viromandues el rhe\% les kimes, devient capricieux. les bourgs traversés sont semés au hasard et leur emplacement parait avoir été déterminé. le plus souvent avant l'époque romaine, par des facteurs nalurels ou humains particuliers.

\section{Le lrace el les bourgs rouliers de la chanssee de Tongres.}

Les mèmes observations ont été faites sur la chaussée de Tongres qui avail recu. en territoire nervien du moins, le mème équipement que celle de Reims (lix. 2). Elle aussi comprenait deux parties fort différentes. Ia première, de bavai au territoire de CortilCoirmond, était d'une parfaite rectitude. L'autre, qui s'étendait jusqu'aux portes de Tongres, avait au contraire un tracé sinueux, attestant qu'il s'agissait "d'une piste de l'àge du fer aménagée pour le trafic romain ${ }^{29}$. in outre, loutes les qualre lieues également.

in Ch. Cholx, op. cil., p. 124-1:27 el M. Hixalit, La voie romaine de Bavay à Reims, dans Bullelin archologique du Comile des Traraur historiques el scientifiques, 19.40-11, 1. 451-169.

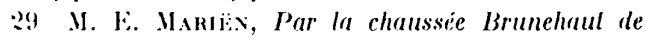
Bavai da Cologne, Musies Royaux d'Art of d'Histoire, ze éd., Bruxilles, 196x, p. d8. 
des relais furent repérés a Goegnies-Chaussee, a Civry, à Vorlanwet z el l'Itinéraire d'Antonin signale l'existence probable d'une mansio a XII lieues de Barai : Vorlgoriacum, que l'on s'accorde à situer à Waudrez ${ }^{30}$. Jusqu'a l'étape suivante. Geminiacum-Liberchies, deux mulaliones ont pu egalement se trouver a Morlanwet\%, déja rilée et a Gouy-lesPiéton ${ }^{31}$, aux conlins des cités des . Verviens et des Tongres ${ }^{32}$.

Les ecarts de quatre lieues entre les relais et, en principe, de douze lieues entre les élapes, qui sont asse\% courts ${ }^{33}$, ont dù lavoriser la rapidité de la transmission des dépèches le long de deux roies administratives et stratégriques de tout premier ordre. Mais le choix de res distances, en territoire nervien du moins, semble avoir été imposé par des facteurs naturels qui ont favorise la concentration en certains endroits de plusieurs établissements administratifs et, notamment, des bureaux de la douane et des relais de la poste. En effet. a des intervalles à peu pres réguliers, la chaussée de Reims lraverse l'Helpe Mineure, puis l'Helpe Majeure, puis la sambre. Par extraordinaire. celle de Tongres rencontre la Trouille a Ciivry. la samme et la Bruille a Waudrez. la Haine a Vorlanwetz le Piélon, enfin. a Gouy. Or. si le porlorium étail percu parfois aux frontiores de la cirilas ${ }^{34}$. le long des principales voies romaines, comme le laissent supposer les loponymes Camaracum-Cambrai et Fisciacum-lusciaux-les-Cateau, sur la chaussée de Vermand, il l'était parlois ausis au passage

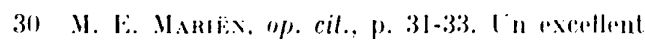
resume des lousailles est fat par $A$. Waxkexile, op. ril., p. $4 \times-49$.

31 .1. E. Mantix, op. cil.. p. 37-3x. Selon linuleur, (in) alurail pu être la premiere mansio du cursus phblicus a partir de Barai. la comparaison entre les

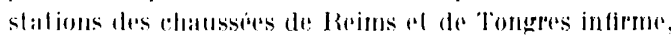
a notre aris, celle hypothese.

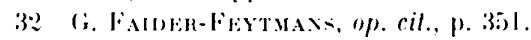

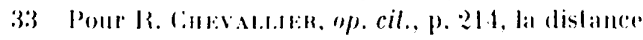
moyenne entre les mansiones on ilapes de muil aurail bo de 30 a $36 \mathrm{~km}$, " ce qui correspond, a ch croire Vegeree Epiloma rei militaris, I, 9, a l'iblape normale d'un ligiomnaire"

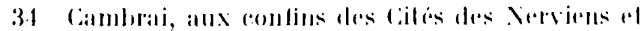

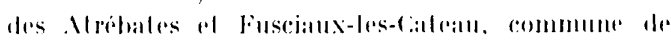
saint-Bening pres de la fronliore antre la lermandois at la Nervie.

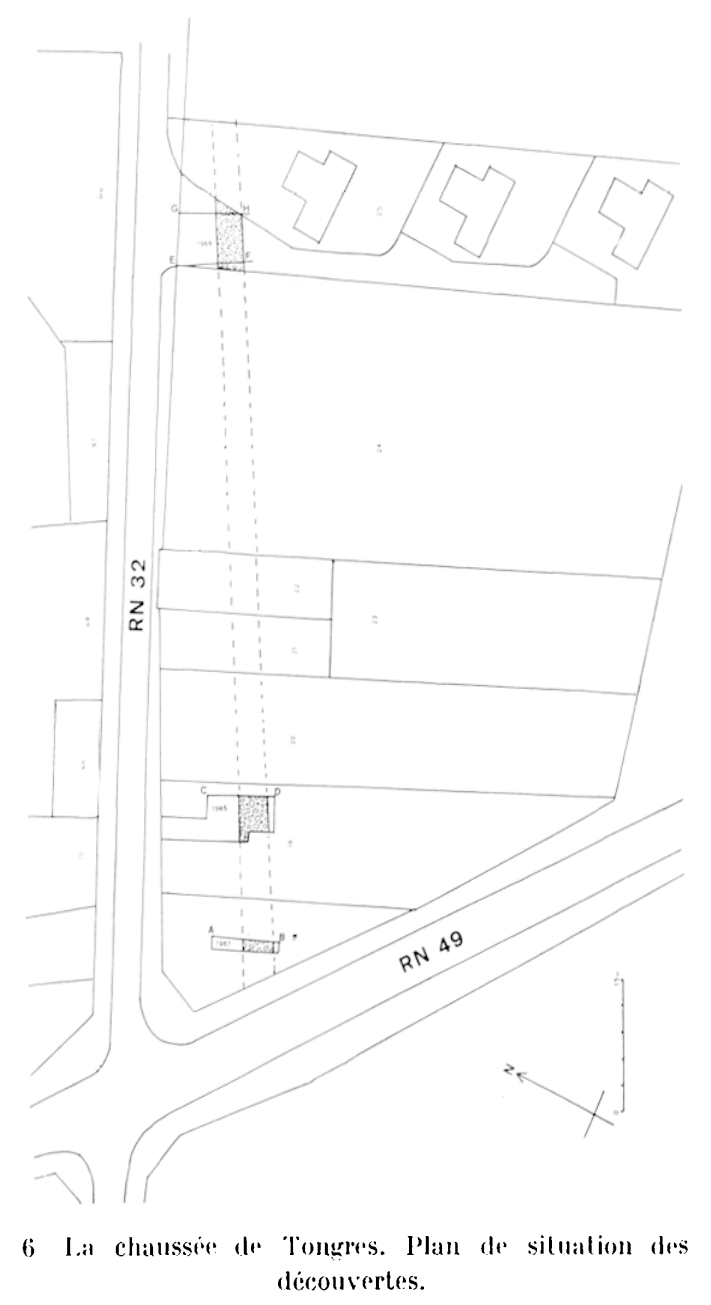

des ponts el des gues romme a FisciacumFuchau, sur la chaussée de Reims.

\section{Struchure de la chanssiée de T'ongres.}

Comme nous pûmes nous en rendre comple dans les parcelles cardastrées sous les nos 18 , 19, 25) (lig. 6), la structure de la chaussée de Tongres présentait avec celle de Reims certaines analogres. Lne fouille exéculée dans la parcelle $n^{0}$ is avec le concours des étudiants des Facultés de Namur ${ }^{35}$ permit de melle au

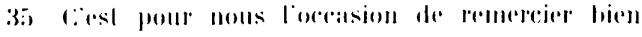

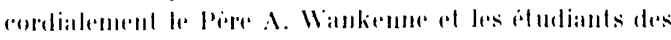
Paculles de Namur qui apportent, depuis 1967 , leur concours erficace a fexploration archeologidure de Baııii. 
jour un troncon urbain de la voie flig. $\bar{T}$, coupe $1-B$. De relle-ci l'empierrement était en grande partie récupéré. Nous pùmes néanmoins observer un hérisson d'éclats de grès, d'une largeur de $6,70 \mathrm{~m}$ el d'une épaisseur variant entre 0,15 et $0,20 \mathrm{~m}$. Au-dessus. des pierrailles de gris schisteux, fortement lassées sur une épaisseur de 0.10 a $0.20 \mathrm{~m}$. formaient une assise que recouvraient deux lits de marne, le premier. de couleur gris bleulé. el le second. de couleur mauve. De la couche de roulement. consistant en un cailloutis de calcaire bleu de $0.30 \mathrm{~m}$ d'épaisseur, l'extrémité norl devenail terreuse. Elle se prolongeait par un troltoir de $0.10 \mathrm{~m}$ d'épaisseur, formé par des tuileaux et des cailloux grossierement lies par du morlier jaune, dont le niveau supérieur était approximativement a la cole 145),70. Ce troltoir, de 3 m de largeur. s'étendait sur un sol de boues yrisàtres auxquelles se mèlaient des os. du charbon de bois el quelques tessons de poteries. Lne recharge de calcaire bleu, dont il ne restail que l'extrémité nord, attestait un glissement. lardif de la chaussee. Un second trothoir, mais d'un mìlre seulement de largeur, bordait au nord le dernier empierrement.

Dans la parcelle contiguë, no 19 , nous avions déjà, en 196\%), observé une autre coupe de la voie (fig. 7. coupe G-I)). Si les trotloirs avient disparu, l'empierrement conservail dans l'ensemble la même structure. I)u hérision de base, la largeur se réduisait à $6,10 \mathrm{~m}$ el celle de la couche de roulement, bombée en son milieu et culminant a la cote 146,201 , a j,30 $\mathrm{m}$ seulement. L'assise de la chaussée était faite avec les mêmes matériaux, mais jetés en tranchée puis recouverts par un lit de marne mauve qui s'étendait probablement jusqu'aux fossés latéraux. Celte couche drainail sans doute les eaux de pluie toul en formant. peut-ètre, une piste cavalière longeant la voie empierrée. Celle-ci, apparemment. avait été rechargée aver un railloulis de calcaire bleu dont les éments ataient bloques sur les bords de la voie par des pierres plus grosses. Au nord romme au sud de ce dernier empierremenl. s'elendait une couche de boues el de gravals s’olevanl jusquan nivean superierur de la route.

Les Iravaux de terrasiement exécutés par le Gaz de France, 59 m environ plus a l'est, dans la parcelle no 25 , permirent dobserver des vestiges différents figr. $\chi_{\text {. }}$. Ieux coupes distantes de 6 m environ montraient que des fossés limitaient probablement l'emprise de la roie. Mais on ne royail. sur la coupe (i-H, qu'un seul fosse au nord. de $0,48 \mathrm{~m}$ de largeur et de 0.2:3 $m$ seulement de profondeur, vers lequel une rouche de marne mauve drainail les eaux de pluie. Dans la roupe b-F au contraire, sobservait au nord une fosie dont le creusement plus tardif avait pu faire disparailre le fossé primitif; mais au sud. sous l'empierrement de lit chaussée, réduit à une couche de gris schisteux lassés dans du sable sur une épaisseur de quelque 0,50 $\mathrm{m}$, se dessinail. entre les roles 145,50 et 146. le profil d'un second fossé mesurant $1.30 \mathrm{~m}$ de largeur et appartenant vaisemblablement a l'état primitif de la voie. De la boue grise, recourrant igalement le sol de limon jaune, le remplissait en eflet ef c'est dans cette couche qu'avaient été jetés en tranchée les matériaux formant l'assise de la chaussée. Ces découvertes font supposer que la roie de Tongres fut, a l'origine une simple piste qu'on empierra par la suite. Cependant, il est curieux de constater qu'aurune des quatre coupes examinées ne révéla l'existence d'une couche de petits silex noirs semblable à celle qui formait, l'empierrement le plus ancien de certaines parties des chaussées de Reims, de Trives el du troncon commun aux routes de Cambrai et de Tournai. Or, de ce dernier, les découvertes de Saint-Waast-La-Vallée précisent nettement. que l'époque de construction se silue entre les années 40 et 50 ap. J.-G. ${ }^{36}$. Iu contraire, lant par les matériaux qui la composent que par sa structure, la chaussée de l'ongres, aux abords immédiats de Bavai, ressemble beau-

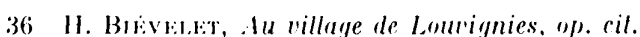
les tessons que nous avons mis an jour at identifiés, (1) dont H. Bievelet fait etat p. 54, peuvent elde dates de la derniere decennie de la premiere moitie du ger siecte. Colle dale semble itre confirmere par les

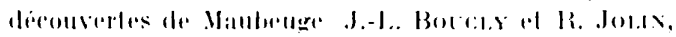

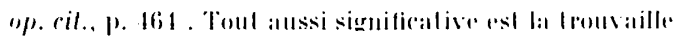
fure mous fimes a saint-llatast. pres de la chatussite romaine, diun follis de tonstance Il imise a drles rers $3: 5-326$ 


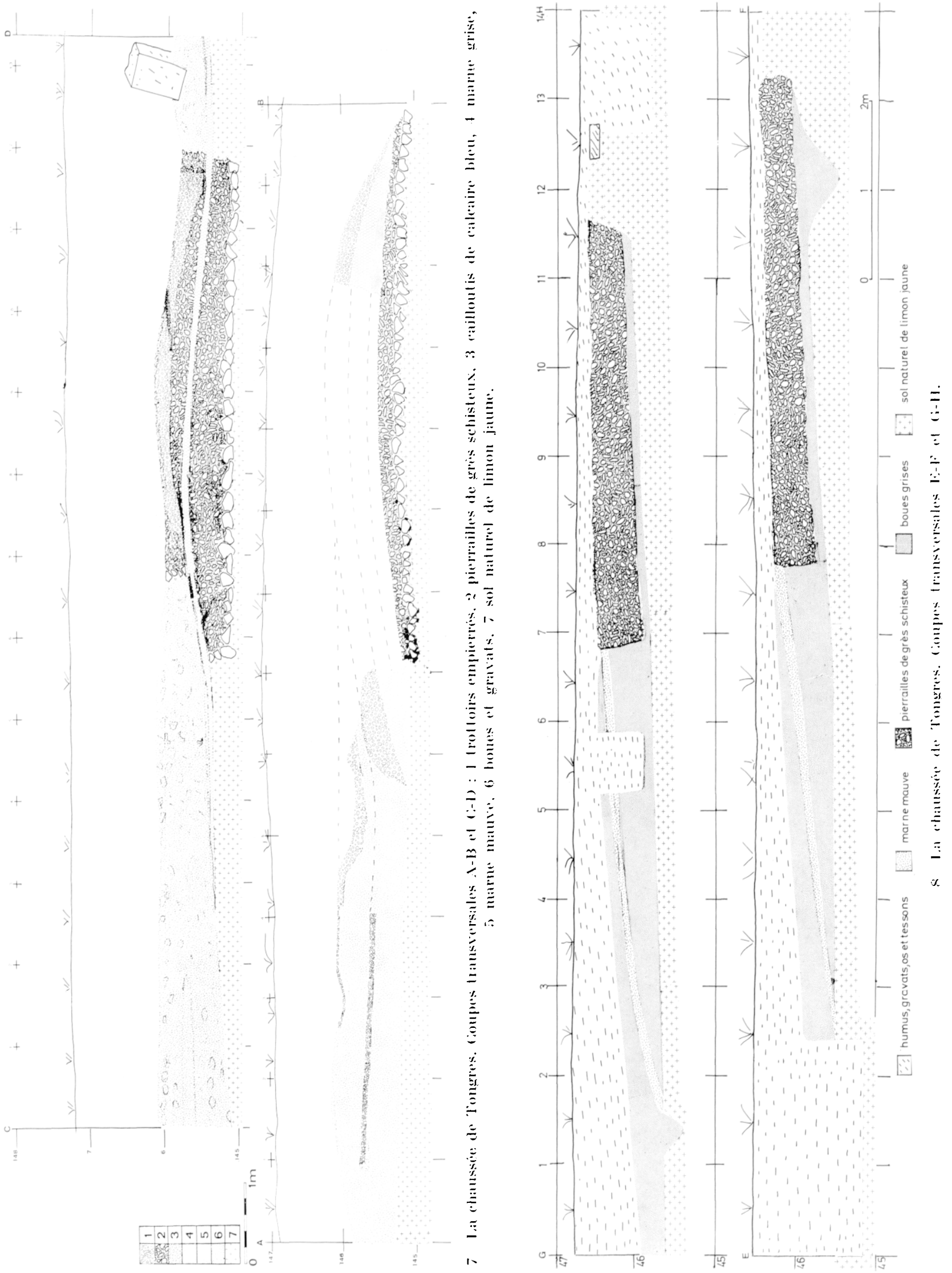


coup au deuxieme élal commu de celle de Reims. dont la chronologie na pu ètre précisée. Quoi qu’il en soit, il est certain que les dernieres recharges de ces deux voies datent du In $^{\mathrm{e}}$ siocle, comme l'attestent les tessons de poleries ramassós dans les rouches supérieures de houes el de gravals. En lout cas. les fragments de colonnes milliaires découverts en 185i] a Godin, en 1870 à Basse-Boulogne, el en 1710 a Vir-sur-disne. le long de la chaussoe de Reims, apportent quelque lumiere sur cette période obscure el indiquent une réfection de la voie sous le rigne de Caracalla, vers 210 ap. J.-C. ${ }^{37}$. Ajoutons entin que le début du III $^{\mathrm{e}}$ sicule ap. J.-C. est également la date assignée au criblore fragment du "milliaire» de Tongres ${ }^{38}$.

Misloire des bourgs remliers des chanssies de Ripims el de Tongres.

()uoi qu'il en soil, gràce à leur équipement et a l'importance de leur trafic. les rhaussees les plus fréquentées allirèrent une partie de la population des campagnes et favorisirent la naissance de bourgs dans une région ou la vie vicinale étail a peu pris inexistante ${ }^{39}$. 1)ans la partie française de la civilas, en effel. lous les rici connus. a l'exception des agglomérations de Famars et de sains-du-Cord, doivent leur existence au réseau routier conçu par Igrippa el développe nolamment sous Claurleto. Iux bourgas précédemment rilés

37 .. L.:ßs:At, sur la découverte d'un milliaire à Gindin, dans Bullelin de ln Commission Mislorique du Nord, XI, 1872, p. 379. (h. liko1x, op) cil., p. 1201-121

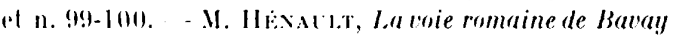
à Reims, op. cil., p. 4t5, n. 1. - E. MaTox, op. cil.,

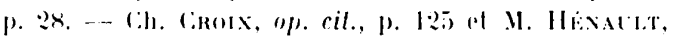
"1). ril., p. 156.

$3 \times$ Pour la hiblographie : 1. Waxkexwe, "p. cil., 1.. 89, 11. 3.

39 Cies observations ont ifle confirmess depuis par les recherches de li. Agache an leicardio : Détection arienne de vestiges probhistoriques, yallo-romains ol módifinular dans le bassin de la somme el ses abords, dans Bullelin de la socislí de Próhishire du .Vord, n" 7 . $197+1,1) . \mid x(1)$

fo Sur a sujel, on consullara utilement $\mathrm{S}$. J. И) I.nve, Clande el Ia Romanisation de la Cianle seplenIrionale, dans Melanges offerls à A. I'iganiol, Ficoll. il laut en ellet ajouter HermoniacumBermerain el Camaracum-Cambrai, traversés par la rhaussée d'Irras ${ }^{41}$.

1 défaul de recherches élendues et programmées. de nombreuses découvertes fortuites el quelques fouilles anciennes ont livré des documents ahondants sur les vici de la chaussée de Reims et permettent d'esquisser l'histoire de res derniers. si monnaies el céramiques prouvent sans conteste leur existence dis la première moilié du ${ }_{1}^{\text {er }}$ siècle ap. J.-C., leur développement date surtout du ${ }_{12} \mathrm{e}^{\mathrm{e}}$ siècle, pendant lequel ils bénéficierent de la prospérité générale de l'Empire. I leurs activités tradilionnelles, qui étaient principalement aqricoles, s'en ajoutirent en elfet d'autres, industrielles el commerciales. I Quartes, par exemple, se concentrirent de nombreuses firmes puissantes, spécialisées dans la fabrication des céramiques communes à pàte jaune, consistant surtout en pelves el en amphores ${ }^{42}$. Je ces ateliers l'aire de dispersion des produits est considérable ${ }^{43}$. D'aulres oflicines fabriquaient égale-

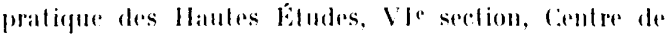
liecherches hisloriques, l'aris, 1966, p. 951-961.

11 II. Hexalta, Bermerain, liaure du lor Tremblin. Recherche sur IIermoniacum, dans Pro vervia III.

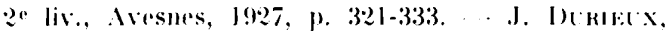
Cambrai gallo-romain el mirouingien, dans . Wimoires

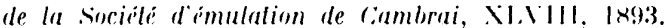

12 Aux decouvertes do depotoirs ol de fours de

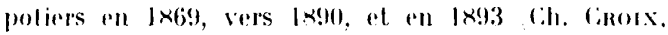
op. cil. p. 109-111, il fant ajouter des trouvailles plus liconles : J. Giracolnt, I proposs dune marque de pelve dícouverle a Lewarde (.Vord), dans lievue du

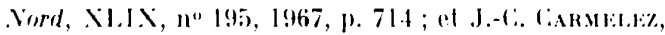
op. cil., I, p. 346 ot H, p. $11-4.4$.

43 II. Hixalig of P. Mancite, les marques de poliers à Bavay, les liles à lail (pelves), daus Pro Vervia, 1, 20 liv. Aresnes, 1923, 1. 115-127; I, 3 of te liv., 1923. p. 207-213; II, Iलr lis., 1924, p. 66-69; II, 2 "liv., 1925, p. 113-122 ; II, 3" Jiv., 1926, p. 193-200); II, fe liv., 1925, p. 257-26.1; III, ler liv., 1927. p. 311-314; 111, 2e liv., 1927, p. 361. - J. Grucolert,

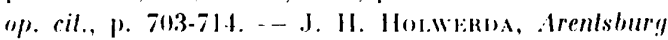
een romeinsch militair oloststation bij Voorbury, levede,

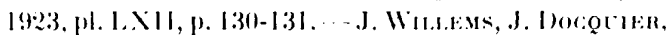

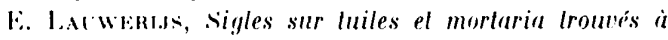
Braines el dma!j de 19.ji it 1961, dans Bullelin du cercle archiologique Hesbaye-(ondroz, II, 1961, p. 30-34.

- Des travaux fondamentaux sur la question ont éti publies recemment par .1 me. K. F. Hanteky, Professem a ITniversilo de loeds, qui, avec beatucoup de compertence, a eludie les margues de pelves tromvers à Bavai : 
ment, a Quartes comme a litroeungt, des matériaux de construction en terre ruite. Les déchels de reux que nous avons observés a Quartes, consistant en luiles a rebords. couvre-joints. briques el boisseaux d'hypocauste, ne le cédaient en rien pour la qualite aux maleriaux des édifices bavaisiens ${ }^{44}$. I Furhau enfin. si l'on en juge par la quantité de scories mises au jour sur le sile mème ou, a proximite, dans l'empierrement de la voie. la siderurgie élait la principale activité artisanale du bourent5. Ainsi, on peut observer qu'aprés le coup d'arrèl donné à l'artisanat bavaisien par la guerre civile de 69 ap. J.-C. el par le développement de l'urbanisation de la ville sous les Flaviens. le chef-lieu de la Cité tendil à devenir un rentre ardministratif, rommercial et résidentiel. The grande partie de ses artivités traditionnelles. celles de la labrication des terres cuiles notamment, fut

K. F. Hantar. La diffusion des morliers, luiles el anlres produils en promenance des fabriques ilaliennes, dans Cahiers alrcheologie subaqualique, 11, 1973, p. 19-60. In., The marlieting and distribution of morlaria, (i.B.A., Restarch leport I0, Current research in Somano-Brilish coarse pollery, 1). 39-5). La figr. 1 monter nolamment la dispersion en Gimmle-Bretagne des prombits signes Adjutor, Marlatis I, Privalus,

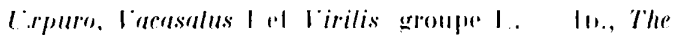
morlarium stamps. Terulamium excanalions. I, dans Reports of the Research commillee of the sinc. "f lntiquaries of Lombon, XXl111, 1972, p. 371-381. 11., The mortaria and lheir origins, dans fifth report on the ercanalions of the Roman fort at Richbormugh. Kent,

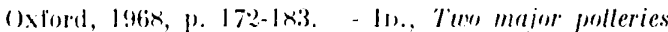
producin! morlaria in the firsl cenlury .1.l)., dans Iroman prollery studies in Brilain and beyomd, Papers presented to J. (Gillan, British Areharological Reports, supplementary series 30, 1977, p. 5-15.

11 Vous anons fail ces decourerles a (ouartes lors du carage de la Sambre en lg6x. Nous avons pu constaler lexistence, antre l'eglise al la riviere, deunt

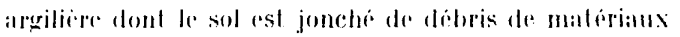
romains all lerre cuile. Catux-ci forment ume rouche asso\% cyatsse visible dans la berge gamehe de la sambre. 1)antres arerilieres semblables se remarequenl non loin de lat riviere, lant à Quarles quatu hameall de.

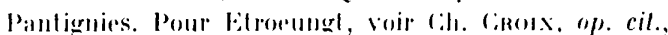

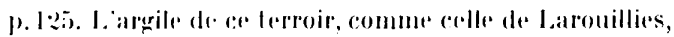

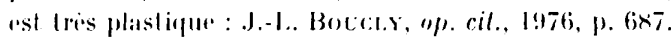

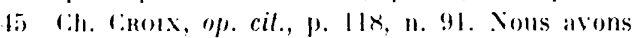
renouvele res observations pris du pont de la vole

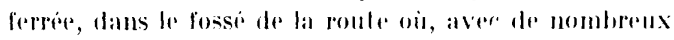
lessons de poleries, nous arons tromve me monmaide

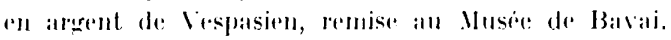

transférée dans d'autres bouraades ou so trouvaient réunies de meilleures condilions économiques : une main-d'ouve et des ressources naturelles abondanles, des moyens de communicalion llusiaux et routiers permettant le transport aisé des marchandises.

De cette prospérite. le dérlin se manifesta brusquement vers la fin du ${ }_{11} \mathrm{e}$ siecle, au rours d'une période de lroubles et d'insécurité dont les trouvailles archéologiques fournissent maintes preuves manifestes ${ }^{77}$. Ln tous as, les grandes officines locales de céramiques a pate jaune disparurent à peu pres completement a retle époque ${ }^{48}$. Durant le mi ${ }^{\mathrm{e}}$ siecle, les bourgades de l'Avesnois connurent, semble-t-il, le mème sort que Bavai. Fortement dépeuplées par les invasions de 204 ap. J.-C., elles furent restaurées en partie pendant l'Empire gaulois comme l'attestent les découvertes de monnaies a l'efligie de Postume, a Furhau, el de Tétricus. a Elroeungt, puis disparurent dans la lourmente de 275. Mais, contrairement a Bavia et a la plupart des bourgs routiers de la chaussée de Tongres ${ }^{49}$, aurune decouverte, a notre

f6 I Omames, notamment, deux voles sto

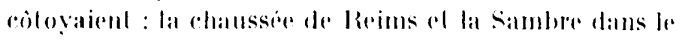
lit de bapuelle on decomvit, rers lx75, les restes d'un deharcadaire: Movox frires, Maulmonl el son abbaye, Haulmont, 1 X95), p. fil. l.es carriers el les chaufourniers

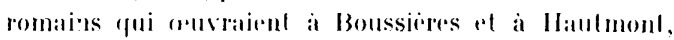
sur la rive galuche de la samber, onl dii égalemenl uliliser la riviere pour le transport d'une parlie des

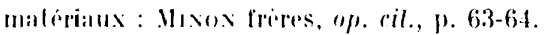

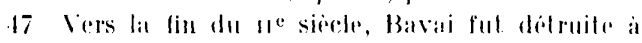

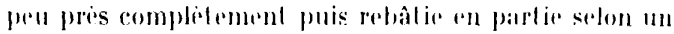

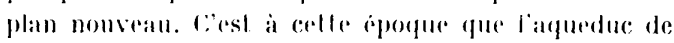

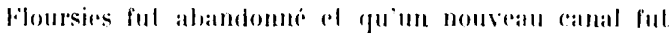
construil, caphant à Échobes et à Écurlin les eaux du hassin superieur du Gimour.

is Jes elablissements ruraux onl pu ble aneantis des cefle epoyur mais une manvaise commissance de la crimique du me siecte, et la dalalion particulierement dillicile de celle-ci a catuse de la disparition presque romplete de la valisselle sigrllere decorie conduisent somvent les chercheurs a all ribuer ces destructions and invasions de 254 ap. J.-6.

19) Les fonilles recentes ont permis de prepiser quapres les premieres mesures de defense improvisees,

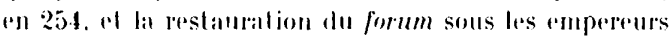
gambos, Bavai disparul rulant que ville sous le conp des imasions de :27) ap. J.- .. An Bas-kmpire. les deax raslella, dont le plus occidental servat au casermement de la trouper, commurent deux phases de construction bien definies, and epoques comstanlinienne (1) vilnomlinimure. 
connaissance, ne prouve la renaissance de ces argolomérations vers la fin du III $^{\mathrm{e}}$ ou all début du rve siecle. Én oulre. nous nous permettons d'y insister, à l'exception d'une monnaie de Constanlin trouvée a dresnes"50. les seuls lémoignages irrécusables d'une présence humaine dans l'Avesnois au we siorleñ lurent découverts par nous dans la vallée de la sambre. a Quartes oì nous trouvàmes quelques lessons de poteries sigillées d'Argonne dans les boues extraites du lit de la sambre. et a Maroilles oì nous venons de reperer. sur le site originel de l'abbaye fondée par saint llumbert vers bio2, les restes d'une rilla encore occupée au Bas-Empire.

Il semble done que. lorsqu'a la fin du In ${ }^{\mathrm{e}}$ ou au début du $\mathrm{w}^{\mathrm{e}}$ siócle, la Cinilas Verviorum fut remplacée par la Cinilas Ciamaracensium, la route de Reims a Bavai fut abandonnée au profil de relle de Cambrai par saint-Quentin. En lout cas. le rhoix du chef-lieu de la nouvelle cilé traduit un mouvement de repli vers le sud et atteste le ròle important que jouail alors l'Escaut comme voie naturelle pour le trafic el les communications52. Quoi qu'il en soit, entre l'kscaut. a l'ouest. et la Meuse, a l'est, formant une ligne d'arrèt en arrière de laquelle la défense fut organisée en profondeur ${ }^{53}$, la chaussée de Tongres a Bavai devint une rocade fortifiee.

5) (il. linolx, op. cil., p. 11.

5) Nous ne pouvons prendre an consideration quelques indications vagues, assorties d'affirmations gratuites comme on en trouve souvent chez les auteurs anciens. Mrsox frieres, op. cil., p. 5.t, par exemple. la localisation a La Fauchelle of à Berlaimont, a l'emplacement de deux ouvages defensifs du Haul Moyen Age, de postes de garde du Bas-Empire relieve, il va sans dire, de la pure affabulation. Toutes ces supputalions permirent d'elayer l'hypothese de la localisation sur la Sambre du port latlache de la Cllassis sambrica.

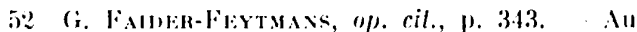
sujel du developpement du lissage de Tournai au Bas-limpire : H. VAX bE WEERn, Het economisch boeilijdperli van Nord- Ciallië in den Romeinschen lija. dans Vededelingen Kon. Flaamse Whad., 1946, p. 1:2.

5) R. Bнtis, Recherches archeologiques sur le Bas-Lmpire romain dans les ciritales Turnacensium. Camaracensium el Tungrurum, l.ouvain, 1975. - 11., La défense du lerriloire au Bas-Empire, dans La Belyique de Cisar a r.lonis. Inossiors de l'. Archeologie, $n^{\prime \prime} \cdot 21,1977$, p. $9 \times-10 \%$. jalonnée par des ouvrages militaires5t. Dans la Civilas C'amaracensium. reus-ci élaient orcupés par des letes nerviens, soldals-paysans dont le préfet résidait a Famars ${ }^{55}$, caslellum qui fermait la trouée entre l'ournai el Bavai, tout en surveillant probablement la traversée de l'Escaut a Trajectum-Trilh-saint-Léger. De res Lites. les trouvailles archéologiques n'autorisent pas à présent à exagérer l'imporLance numérique. si leur présence est altestée a Famars et dans ses environs, a Valenciennes ef à Bermerain notamment ${ }^{56}$, a Bavai, ils paraissent former un ilot de peuplement dans une région redevenue presque déserte ${ }^{57}$. Dans

5) J. Vt:RTwis, (Jueligues aspects de la romanisalion dans l(Onesl du l'ays Gianmais, dans IJelinium, III,

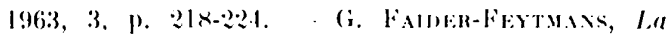
fronliere du Nord de la Cianle sous le Bas-Empire, dans Milanyes offerts ì .I. Marouzeau, less Belles Leftres, Paris, 191x, p. 161-172. - sur les ouvages fortifies de la chausser de Tongres, une bibliographie ahondante ust rassembler dans Cartes archiologiques de la Belgique l-:, op. cil., 2 Divisions administralives, roules, aggloméralions, ay!glomeralions forlifices, bur!fi el castella sous le Bas-Empire, p. 15-28. - J. Ml:RTws, oudenturg el le lilus saronicum en Belyigue, dans Kelinium, II, 1962,

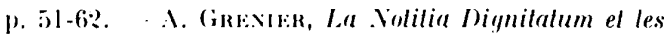
fronlieres de l'Esl el du Nord de la Cianle, dans . Mélan!jes

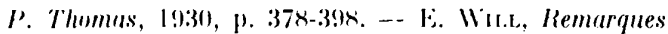
sur la fin de la dominalion romaine dans le . Vord de la liaule, dans Revue du .Vord, XI.1III, 1966, p. 517-531.

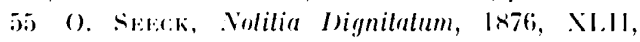
p. 217 .

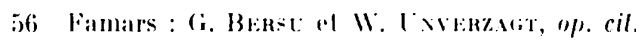
Bermerain : II. Hevalit, op. cil., p. 333.

Valenciemes : cimeliere sainl-hoch, sur la rive droike le l'Escaut J.-C. Canmet.r., "p. cil., p. 311.

if loes lombes du Bas-Empire furent decouvertes a Bavai même el a la peripherie de la ville antique, sur 10 terriloire des communes de Louvignies trouvailles inedites de J. Ouevy, de IJoudain-I.ez-Bavay explorations inédites de .l. Henault, en 1937 et d ll. Bievelet. all 1939 .l, probablement, de Buvignies. J. DI: BAst, I'remier supplíment au recueil dantiquités romaines el gauloises, (iand, lso9, p. 62-64, signale en effol la mise alu jour "a un quarl de lieue de la porld de Gomerie" d'un tombeall remarquable contenant

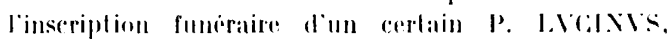
seriniarius de son elal, decede dans sal 4te annere. sous le sixieme consulal de limperene Honorius. \&. Wrosanoms, Nolice sur les monumenls ipigraphiques de Banai el du musée de Monai, Jou:ai, IX7.4. p. \$1-53, range l'inscription de IACilovs parmi les monuments faux ou suspects. Enfin, un pen plus au sud, sur le territoire d'obsies, une lombe, dont la 

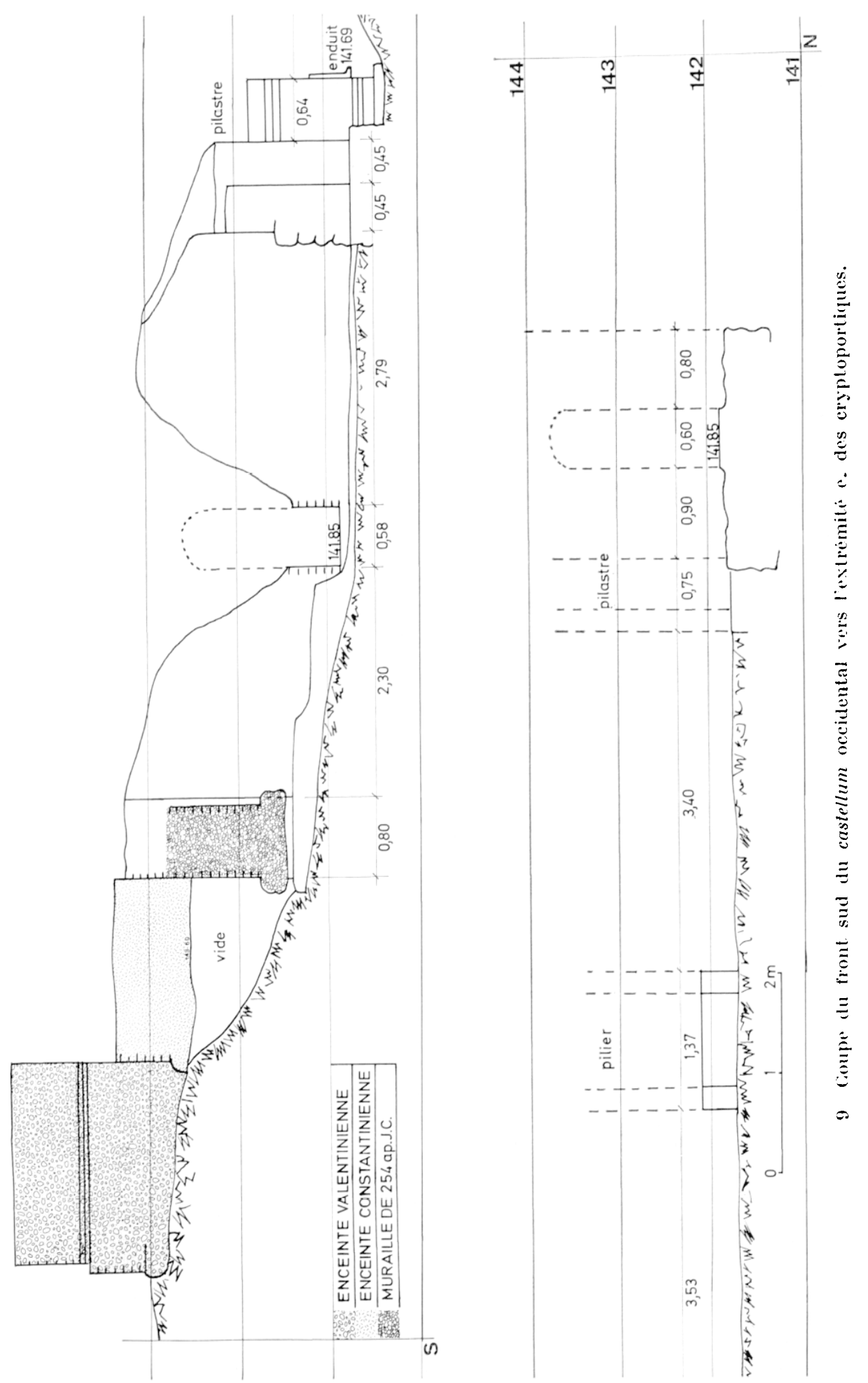


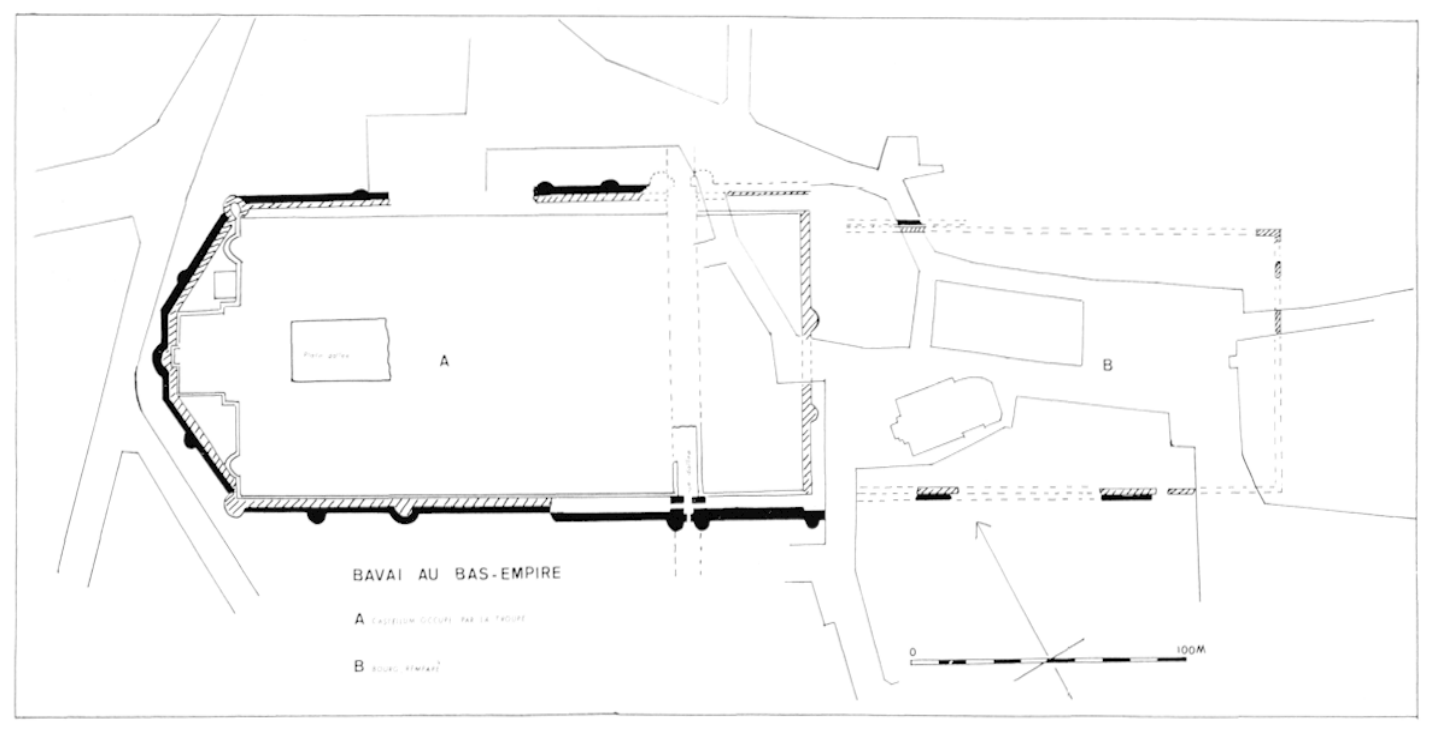

10) Bavai au Bas-limpire. Plan de situation des deux caslella.

le Cambrésis au contraire, les découvertes anciennes el les prospections modernes, celles de Bourlon, de Naves, de Proville, d'()isy. de Rumilly, de Masnières, de Niergies, de séranvillers, en particulier ${ }^{58}$, altestent la

datation tardive nous paraît suspecte, fut exploréc to 12 avril 1928 : R. Terassse, Complement au répertoire des fisemenls archóologiques de l'arrondissement d'Avesnes el des cantons limilrophes, dans Mémoires de la Sociŕli d'Archónogie el d'Itisloire de l'arrondissement d'Avesnes, XVI, 19.10, p. 22.1. On le conslale : laire geographique de ces trouvailles est tres limilio. Ce fait modifie de facon importante la carte du secteur nord-onest de l'Fimpire romain aux $w^{\mathrm{e}}$ et $w^{\mathrm{e}}$ siecles, dessine par J. Mertens dans Oudenburg..., op. cil., p. 6:.

is Nous devons ces renseignements a lobligeante. anitie de J.-(. Carmelez (op) cil. I, p. 315-399. Cambrai : J. Dtratcx, op. cil. -- Bourlon : L. (inatvix it A. TLrras, Recherches archéologiques récentes dans le Cambrésis, dans Revue du Vord, I,I, 202, 1969, 1. 375-379. - Naves et Proville : A. Vaxuenschtidex, Le Cambrisis gallo-romain, nouvel étal des recherches, dans Revue du .ord, I.VI, 223, 1974, p. 533-56x. Marcoing : sépultures a incincration trouveses de 1926 a 192x au lieu-dit la Brique-Moderne: Ch. Gonos, Annales de la Soc. Géologique du Nord, I.III, 1928, p. 295. - Haussy : découvertes de monnaies of de ceramiques de l'epoque de constantin; M. Cinnts, Objels el monuments gallo-romains découverts sur l'emplacemenl dillermoniacum, dans .Mémoires de la Société d'Émulation de Cambrai, I.X, 1897, p. 70-71. pérennité de la vie rurale dans un riche terroir où la population jouissait d'une sécurité relative. Dans l'étal présent de nos connaissances, il semble done qu'entre la région située a l'ouest de la Meuse ou le système défensif avail élé échelonné en profondeur et les terres a blé du Cambrésis, s'étendait une zone de sécurité d'une soixantaine de kilomètres, permettant peut-être à des éléments mobiles de poursuivre éventuellement les bandes ennemies.

Ainsi donc, les roules de Reims el de Tongres, qui avaient été à l'origine des instruments de

Iwy : trouvaille d'une monnaie de Constance II; J.-C. CARMEI:z, op. cil., I, p. 252. - La datation du cimetiere de Ilontay est sujelte à caution. Toutefois, la mise au jour de plats "en aluminium" (sic), 'ntendons, probablement, en métal étamé et de deux monnaies en or de Valentinien et de Valens paraît confirmer l'existence d'une occupation romaine tardive dans la région du Cateau : Ch. Conorx, Le Cateau el sa région, note sur quelques découverles, dans Pro Nervia, II I, ler liv., Avesnes, 1927, p. 297-300. - Sur l'implanlation des Laeti en Belgique: J. BREukr el H. RoOsexs, Le cimeliere franc de IIaillol, dans Annales de la Soc. Arch. de Namur, XIVIII, 1956, p. 171-373 et S. J. DE

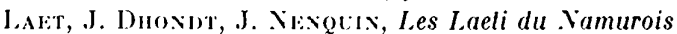
el l'origine de la civilisation mérovingienne, dans Eludes dilistoire et d'Archónglogie namuroises dédiées à F. Courloy, Namur, 1952, p. 159-172. 
pacification des pays conquis, connurent au Bas-Empire des destinées fort différentes. Si la première, en effet, paraît avoir été volontairement désertée, la seconde devint, au contraire une ligne de résistance jalonnée par des garnisons retranchées. I a civilisation romaine qui, en se diffusant le long des grandes chaussées ${ }^{59}$, avait rayonné de Bavai, n'y

59 .I. Vaxdshorvix, De terra sigillata le Tongeren. De ilalische terra sigillala, dans IIelinium, VII, 1967, fasc. I, p. 32-64 et, surtout, fasc. 3 , p. 193-228. survécut au Bas-Empire que grâce à l'aménagement militaire d'une partie seulement du réseau routier, mais repliée à l'abri précaire de murailles défensives (fig. 9), dans l'étroit espace qu'occupaient les deux castella ${ }^{60}$ (fig. 10).

Jean-Iouis Botcis.

60 F. WiLt, Les enceinles du Bas-Empire à Bavay, dans levene du Vord, LIV, 1962, p. 391-401. -- On consultera egalement les comptes rendus des fouilles publiés par II. Bínventet dans la Revue du Nord, XI.VI, 1964, p. 183-205; XLVIII, 191, 1966, p. 557576 ; LVI, 223, 1974, p. 455-459. 